\title{
Structural Optimization of Composite Wings in an automated Multi-Disciplinary Environment
}

\author{
Tobias Bach $^{1}$ and Sascha Dähne ${ }^{2}$ and Lars Heinrich ${ }^{3}$ and Dr. Christian Hühne ${ }^{4}$ \\ German Aerospace Center (DLR), 38108 Braunschweig, Lower Saxony, Germany
}

\begin{abstract}
This paper presents a structure design and optimization module, developed for the application inside multi-disciplinary optimization process chains. The loads necessary for sizing and optimization are calculated in CFD or aeroelastic calculations and applied on a Finite Element Model that represents all primary structural elements of a wing. The FE model is created automatically from a parametric geometry description. The deformations and inner loads of the wingbox are calculated via linear static FE calculations; geometry and loads are provided to an external sizing tool. Each component has a set of design variables with discrete design points which are permutated to get the component's design candidates. A set of failure criteria is used to size the structure which can be made of composites or metal. The methodology is applied to the optimization of a forward swept composite for a short range aircraft and a design study comparing different stringer types and their influence on mass and structural deformation of the wing is performed.
\end{abstract}

\section{Nomenclature}

$\begin{array}{ll}\Delta m & =\text { change in mass between two iterations } \\ \Delta s & =\text { stiffener pitch in stiffened panels } \\ A M I F & =\text { Aerodynamic Mesh Interface File } \\ b d f & =\text { Bulk Data File, Input file for MSC Nastran } \\ C A D & =\text { Computer Aided Design } \\ C F D & =\text { Computational Fluid Dynamics } \\ C P A C S & =\text { Common Aircraft Configuration Scheme } \\ C S M & =\text { Computational Structure Mechanics } \\ c_{m} & =\text { moment coefficient } \\ C_{p} & =\text { pressure coefficient } \\ F E M & =\text { Finite Element Method } \\ L L & =\text { Limit Load } \\ M D A & =\text { Multidisciplinary Analysis } \\ M D O & =\text { Multidisciplinary Optimization } \\ M o S & =\text { Margin of Safety } \\ m_{F} & =\text { fuel mass } \\ n_{z} & =\text { load factor } \\ S B O T & =\text { Sizing Robot } \\ S F & =\text { Safety factor, related to load level } \\ S M T & =\text { Shear-Moment-Torsion } \\ U L & =\text { Ultimate Load } \\ W_{\text {struct }} & =\text { Structure Mass }\end{array}$

\footnotetext{
${ }^{1}$ Research Engineer, Institute of Composite Structures and Adaptive Systems, Tobias.Bach@dlr.de

${ }^{2}$ Research Engineer, Institute of Composite Structures and Adaptive Systems, Sascha.Daehne@dlr.de

${ }^{3}$ Research Engineer, Institute of Composite Structures and Adaptive Systems, Lars.Heinrich@dlr.de

${ }^{4}$ Head of Department Composite Design, Institute of Composite Structures and Adaptive Systems, Christian.Huehne@dlr.de
} 


\section{Introduction}

$\mathrm{M}$ ULTIDISICPLINARY Optimization is a key factor for obtaining efficient aircraft designs with respect to aircraft requirements like aerodynamical, structural or cost performance. ${ }^{1}$ In industry and research several Multidisciplinary Optimization (MDO) processes have been implemented to solve the problem of possibly differing optima of each sub-field ${ }^{2-4}$. Nevertheless a computationally efficient process is necessary to obtain results in an acceptable amount of time. ${ }^{1}$ This requires a well-defined optimization framework as well as efficient tools inside this framework used for the disciplinary calculations. These requirements have to align while still using models with a fidelity that is high enough to represent the complexity of the discipline problems. In order to obtain accurate results for mass and stiffness all influences on these values have to converge with the FE model, which determines its fidelity. This is especially important for wings due to the fact that its deformation is used to modify CFD meshes in aero-structure coupled calculations. The pressure distributions represent the outer loads and an error in them would lead to an error in the inner loads as well. The result would be a different optimization result. On the other hand a model with a high fidelity requires more time to create and to calculate the deformations and forces so the fidelity should not be set to high in order to save time during the MDO process. In this paper a sizing and optimization methodology for a wing in a multidisciplinary process is presented and demonstrated. The used case is a forward swept composite wing is presented.

Because of the potential weight savings and their possibilities to gain tailored elastic properties, i.e. for twist control of laminar wings, composites are more and more used for primary aircraft structures. Any newly developed structure optimization module for MDO applications should therefore be capable of using composite and metallic materials. This will provide the possibility to evaluate a wide range of configurations including the component's design concept itself and different materials for each component.

\section{MDO Architecture at DLR}

The use of Multidisciplinary Analysis (MDA) and MDO has a long standing tradition at DLR. In several projects like MDOrmec ${ }^{4}$ or VAMP, different multidisciplinary optimization processes have been developed. In these projects wings or fuselages based on metallic designs are optimized from aerodynamic and structure mechanical point of view the loads were usually calculated for a given set of load cases with CFD-CSM coupled calculations. CAD geometries were used as geometrical representation. In the used sizing module named "SBOT", a Matlab-based sizing routine, different critical criteria like yield strength of the probed materials and global stability constraints for the different load cases were evaluated in a fully-stress design approach. An optimization of stiffened panel design concepts was not possible. The mechanical properties of a chosen stringer were smeared into the panel properties with an additional layer with equivalent material properties so the longitudinal stiffness of the panel was kept constant. During optimization this layer thickness was modified and the difference in layer thickness is used to scale the stringer web thickness. This approach was used because a retransformation from a smeared stringer to a discrete stringer is not a unique problem. Being used as a tool for preliminary design, the execution time is crucial, therefore the composite materials were treated with smeared properties, and so were stiffeners. Smeared properties allow a fast optimization but for composites a modification of the layup and the stacking sequence influence on bending stiffness cannot be considered. This can lead to structures where the stiffness is treated very conservative and the structure mass is either significantly to low or too high, depending on the used criteria. From the aerodynamics side, optimizations of the aerodynamic profiles and the wing planform (sweep angle, span, etc...) were performed.

In current projects like AeroStruct or Digital- $\mathrm{X}^{7}$ it is the goal to optimize composite wings or complete aircrafts using metallic and composite materials. In addition to previous projects more structural elements like stringer and spar caps will be considered. More design parameter and also design concepts of a wing will be optimized. In addition to the optimization of the structural parameters of the elements the developed optimization framework is also able to change the used type of stringer of a stiffened panel or the number of ribs in a wingbox. Depending on the project, the loads are calculated for a given set of loadcases from the v-n-diagram or determined by the process itself. If a wing is optimized it is possible that the critical loadcases change due to modifications of the aerodynamic layout. This can be considered if the critical loadcases are chosen by the process itself. In order to obtain more realistic results, structural concepts and composite laminates cannot be treated with smeared properties anymore.

In order to create a common parametric description for disciplines involved in a MDO process, the Common Aircraft Configuration Scheme (CPACS) has been initiated inside DLR. The CPACS description is under development in order to achieve the possibility to store more information for the different disciplines. Inside the CPACS file, which is xml-based, all information about an aircraft can be stored. For wings information about aerodynamic profiles, their location and orientation in a global aircraft coordinate system are stored. In combination 
with geometry libraries, the model generators of the different disciplines are able to use the exact same geometry. The need to generate files in a CAD system with their own geometry representation is not necessary anymore. An elaborate post processing of the CAD geometry before creating a finite element model is not necessary anymore. Furthermore deviations in geometry during its import and post processing can now be avoided. In addition to the outer aerodynamic contour information about structural elements like spars and ribs and their locations, used materials or design concepts are stored. Also it is possible to add information about load cases, flight missions or costs can be stored.

An additional advantage of the common parametric description is the reduction of interfaces between disciplinary software, see Figure 1. Instead of defining interchange files for each discipline and software, the results of a calculation are written back into the CPACS file and then used by other disciplines.
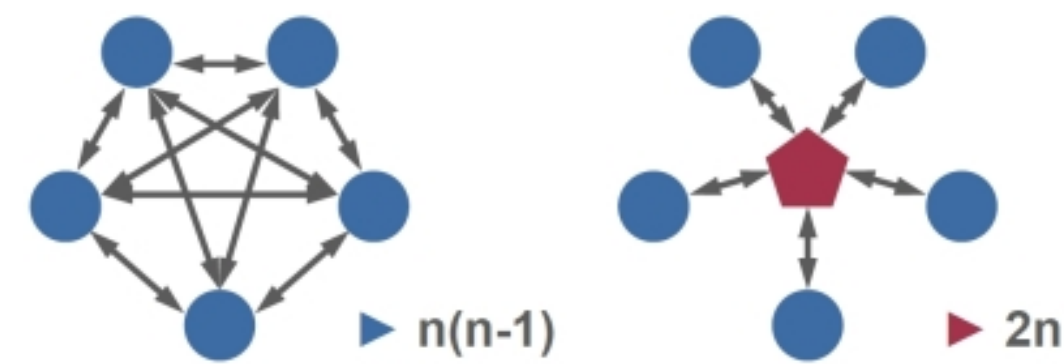

Figure 1: Interfaces in MDO Design Process without (left) and with (right) CPACS $^{5}$

Based on the work in previous projects and the requirements on the structural optimization in the current ones, the development of a new structural design and optimization module was necessary. The goal was to develop a software module that is usable in various process chains and also as stand-alone software if model and loads already exist. The software module should be capable to size and optimize aircraft wings as well as rotor blades or fuselage panels. A modular build-up of the software is necessary so a sizing can be performed with existing failure criteria used in the industry or newly developed criteria. Furthermore the user should have the ability to switch different criteria or required safety factors on and off in order to get information about their influence on design and mass. Of course, the capability to optimize metallic parts should not be lost. Therefor the usage of composites and metallic materials must be enabled in the software. As an important improvement composite layups and stringer in stiffened panels are treated explicitly. For composite materials this is important because manufacturing constraints have an important influence on the laminates stacking sequence. The stacking sequence plays an important role on the bending stiffness of the panel. Not considering this leads to different material thickness if buckling criteria are evaluated during the optimization. Stringers are treated explicitly so local buckling criteria and stringer crippling can be considered.

\section{Structural Optimization Module}

For a useful structural optimization a model that allows the calculation of realistic deformations and loads is necessary. The model must include all load carrying elements in a detailed representation so a realistic load distribution between the elements is possible to calculate. For this purpose, an existing Finite Element Model generator is enhanced for the process so this requirement is met. With this improvement wing cover, spars ribs and spar caps can be modelled and metallic or composites can be used as material. The model generator is coupled with the overall MDO process and with an FE-solver and HyperSizer ${ }^{6}$. HyperSizer is a software used for sizing and optimization of stiffened and unstiffened panels and beams.

\section{A. FE-Model}

The parametrization of the wing with the CPACS file allows great flexibility in terms of geometry and structural layout of the wing. The model generator processes the CPACS information and creates an internal object model where the geometry and properties are stored for each component. The geometry and mesh are created using an automatic FE-model generator. The mesh size can be chosen by the user with respect to the use case. An exemplary wingbox is shown in Figure 2 where a coarse mesh is used.

\footnotetext{
${ }^{5}$ ILT “CPACS Common Aircraft Configuration Scheme” http://cgi.tu-harburg.de/ iltwww/de/cpacsrce/cpacs/cpacs.html
} 


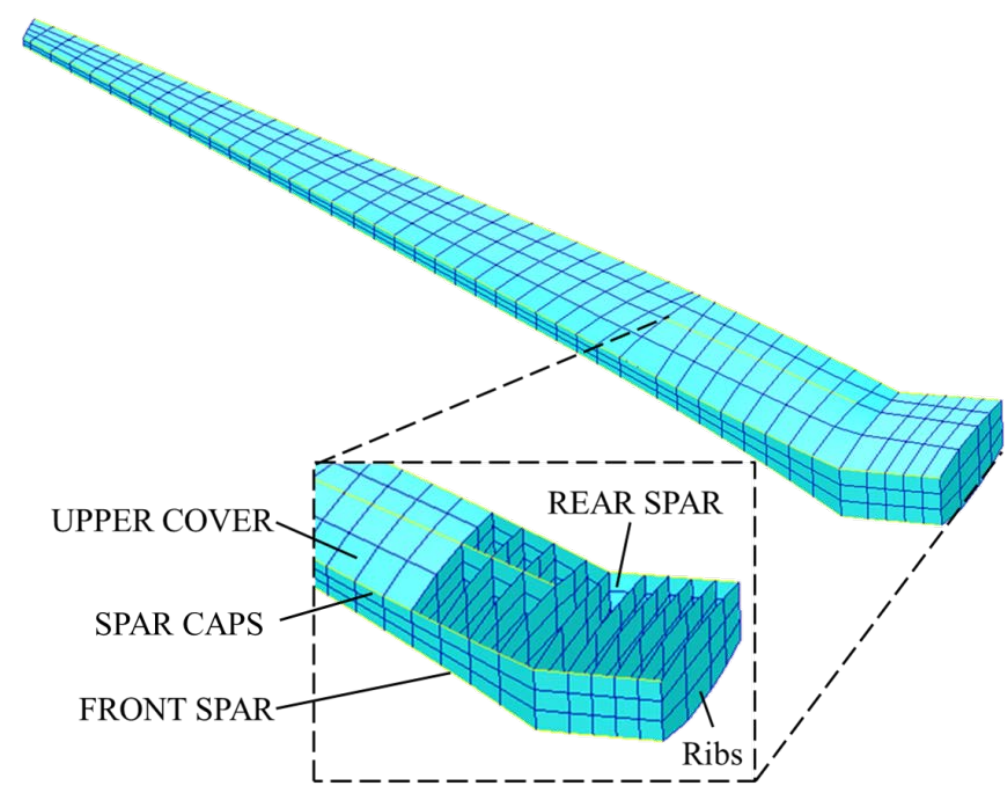

Figure 2: Example wing from model generator with coarse mesh

As structural components the upper and lower cover, ribs, front spar, rear spar and a middle spar are discretely modelled in this example. The spars are provided with bar elements to represent the spar caps. The number of components is independent from the number of information about the structural components in the CPACS file. The model generator automatically cuts in the wing covers and spars position into components at each rib position. For example it is possible to define only one upper cover skin for the complete wing in the CPACS file (which means less work in the beginning) while nevertheless be able to get more than one thickness for the wing covers and spars.

In order to get more versatility, Nastran and Ansys models can be created.

\section{B. Boundary Conditions}

The boundary conditions applied on aircraft wings depend on the process the optimization module is used for. If the wing is optimized without the fuselage a symmetry condition is applied in the middle of the center wingbox, where displacement and rotation is fixed. In addition a vertical displacement at the wing - fuselage interface is prohibited. The boundary conditions are shown in Figure 3.

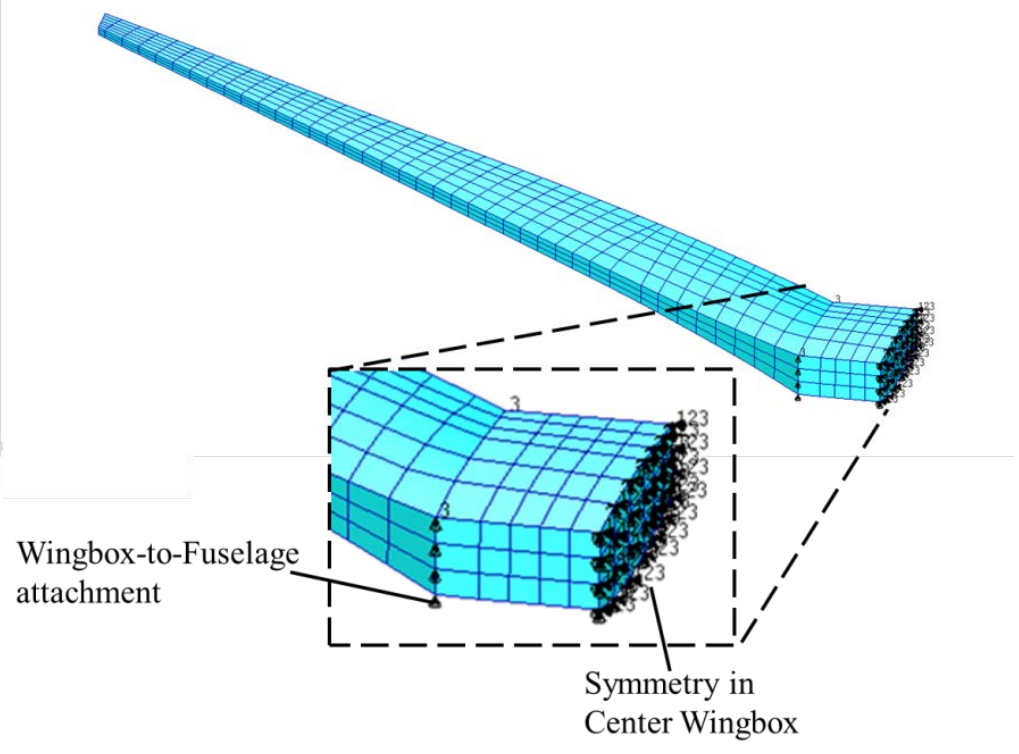

Figure 3: Boundary Conditions of Wingbox

American Institute of Aeronautics and Astronautics 
In case a whole aircraft is optimized the wing and fuselage are connected with MPC elements. The symmetry condition at the wing symmetry plane is not necessary anymore because both wings are modelled and the complete aircraft is pinned at the fuselage rear end to avoid rigid body motion.

\section{Load Introduction}

For loads introduction different methods can be used, depending on availability in the process chains. If shear, moment and torsion curves exist, they can be applied as Shear, Moment and Torsion; "SMT-loads". The shear, bending and torsion curves are post processed calculated to cut-loads at the rib stations along a virtual axis, and nodes at the intersection of this axis and the ribs are connected to the structure with multi-point-constraints; in Nastran RBE3 elements are used. This is shown exemplarily in Figure 4.

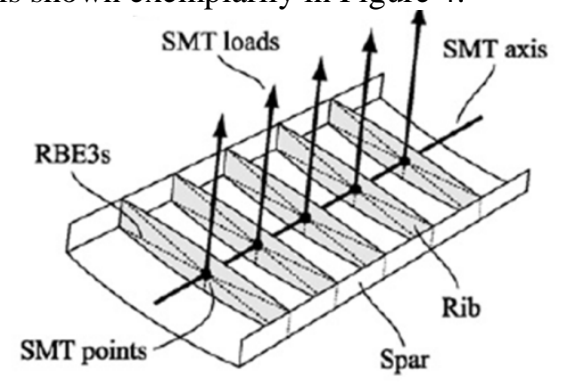

Figure 4: SMT loads ${ }^{13}$

Another possibility is the usage of CFD pressure distributions. As interface format, an "Aerodynamic Mesh Interface File" file (AMIF-file) is used. The $c_{p}$ and $c_{m}$ values are post processed to generate force and moment cards for each load and are included in the Nastran bdf.file.

During an optimization the process is not limited on using one type of load introduction. For every loadcase that exist in the CPACS file, loads are calculated and applied on the model depending on the load cases classification (CFD or non-CFD loadcase). Loads from engines or landing gears are smeared into SMT-loads. In a future version they can be introduced directly via rod elements.

Independent from the loads introduction method, displacements and element forces are calculated with linearstatic FEM. Afterwards they are used for sizing and optimization.

\section{Failure Criteria}

For sizing and optimization a broad variety of failure criteria can used depending on the application scenario. In addition to global stability, laminate based strength calculation, ply-based strength, local buckling, crippling and column buckling can be considered. For all criteria a Margin of Safety (MoS) is calculated with analytical methods. For the usage in MDO applications, a set of default criteria is defined. Along with this their required MoS, safety factors and the load level where they have to be evaluated for are also defined. All criteria can be switched on and off and Load Level, safety factor and required MoS can be modified to see the influence of failure criteria and the structural design. The defaults for composite stiffened panels are shown in Table 1. As load level, Ultimate Load (UL), Limit Load (LL) or a factored Limit or Ultimate Load can be chosen. 
Table 1: Default failure criteria for composite panels and spar caps

\begin{tabular}{|c|c|c|c|c|}
\hline Failure Category & Criterion & $\begin{array}{c}\text { Default Load } \\
\text { Level } \\
\end{array}$ & $\begin{array}{c}\text { Required } \\
\text { MoS }\end{array}$ & Applied on \\
\hline \multirow[t]{2}{*}{ Global Buckling } & $\begin{array}{l}\text { Panel Buckling Compression } \\
\text { and shear , uniaxial or biaxial } \\
\text { and with shear interaction, } \\
\text { Curved or Flat Panel }\end{array}$ & UL & 0 & Panel \\
\hline & Column Buckling & UL & 0 & Stringer \\
\hline \multirow{3}{*}{ Local Buckling } & Local Skin Buckling & LL & 0 & Skin \\
\hline & Local Stringer Web Buckling & LL & 0 & $\begin{array}{l}\text { Stringer Web, } \\
\text { Stringer Flanges }\end{array}$ \\
\hline & $\begin{array}{c}\text { Composite Crippling (MIL- } \\
\text { HDBK-17) }\end{array}$ & UL & 0 & Stringer \\
\hline \multirow{3}{*}{ Ply Strength } & Max Strain 1 direction & UL & 0 & Skin, Stringer, caps \\
\hline & Max Strain 2 direction & UL & 0 & Skin, Stringer, caps \\
\hline & Max Strain 12 direction & UL & 0 & Skin, Stringer, caps \\
\hline \multirow{3}{*}{ Laminate Strength } & CAI & UL & 0 & Skin, Stringer, caps \\
\hline & TAI & UL & 0 & Skin, Stringer, caps \\
\hline & SAI & $\mathrm{UL}$ & 0 & Skin, Stringer, caps \\
\hline
\end{tabular}

The default strength criterion for composites is always the max strain criterion because of the multi-step process that is used to determine the composite layup. Before a discrete layup is calculated for each component a smeared laminate assuming smeared elastic properties and a laminate thickness is calculated. Hereby a stacking sequence is unknown and so a layer wise strength evaluation is not possible. For a downstream detailed analysis more criteria like Tsai-Wu, Tsai-Hahn, Puck, Hashin, etc. can be activated by the user. The equations are described in references 8-11.

For metallic parts the von-Mises stress is used to evaluate the strength of the part. As allowable, yield strength is used by default. If a fatigue loadcases is present the required MoS can be increased.

\section{E. Sizing and optimization}

The goal of the structure optimization is to minimize the weight with respect to a set of failure criteria.

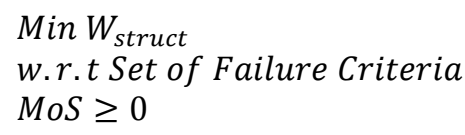

For the sizing and optimization itself, the software HyperSizer plays a key role: the sizing and optimization of the structure is performed with it. In HyperSizer design concepts for stiffened and unstiffened panels are assigned to the optimization regions, which are the property sets from the FE-model. The FE results in form of displacements and element forces are post processed and component loads are calculated for each optimization regions. The embedding of the sizing and optimization module in the global process is shown in Figure 5. 


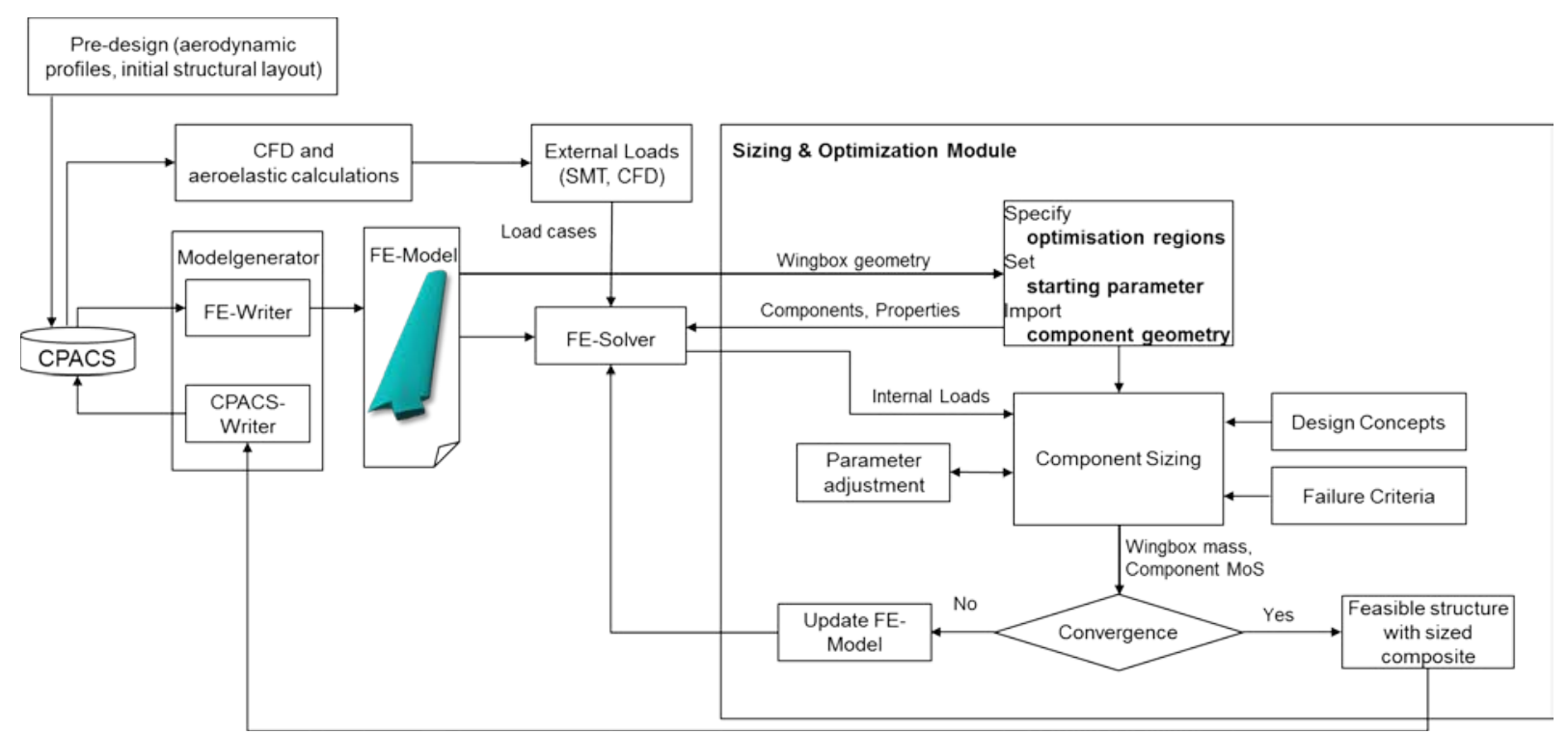

Figure 5: Sizing \& Optimization in global context, one iteration of global process

After importing FE model and FE results, components and assemblies are defined inside HyperSizer. Independent from the finite element solver, each property is defined as a component. One design concept is assigned to each component for the optimization. For 2D panels, stiffened and unstiffened panel concepts can be investigated. Thereby it is not necessary that stringers are modelled discretely in the FE-model. HyperSizer has an internal object model where the component loads are distributed to skin and stringer for each component. This feature allows a much simpler modelling of the wingbox. Because the stiffeners are considered inside HyperSizer they are still part of the optimization even though they are not modelled discretely. Their influence on mass and stiffness is not neglected. Currently, I- and Tee-stiffened panels are used during optimization, and also unstiffened, solid laminates.

The components of a part are combined to an assembly in HyperSizer. An example for an assembly is the upper cover or the front spar of a wingbox. On assembly level manufacturing criteria like symmetric and balanced laminates are applied.

In contrast to other tools ${ }^{2}$, skin and stringer of stiffened panels are not optimized separately. Inside HyperSizer the calculations are performed on a super-stringer like object, exemplary shown in Figure 6, showing all design variables for I-stiffened panels.

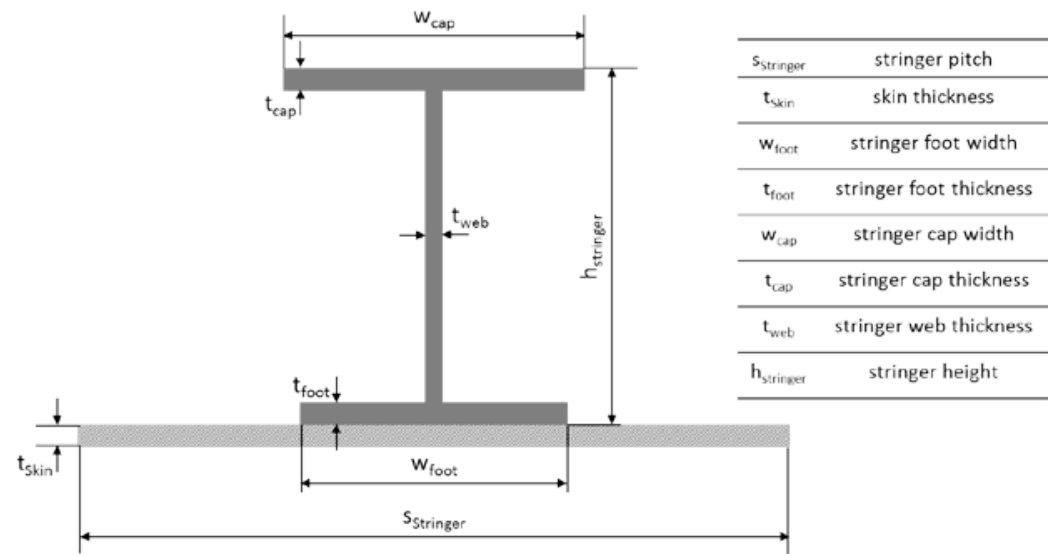

Figure 6: Superstringer-Object in HyperSizer with I-stiffener design variables, no curvature shown

For each design candidate the loads of skin and stringer are recalculated, depending on the stiffness difference between the parts. The allowables for the applied failure criteria are also recalculated for each design candidate if necessary. An example for that is local stringer web buckling if the web height or the ply distribution changes. During the optimization the parameters describing the structural behavior for skin and stiffener like material (respectively ply distribution), stiffener type or pitch are varied for each component, whereas each parameter has a 
set of discrete design points. The number of design candidates is given by the number of the design point's permutations. With this strategy and optimum skin-stringer combination for each component (skin and stringer laminate, pitch, stringer height) is calculated.

The properties of the laminates are calculated in a two-step process, where in a first step the number of plies of the laminates of each optimization region, depending on ply distribution and target thickness and its parts are calculated. The ply thickness of the used material is known. In a second step a set of discrete layups is created and the combination with a minimum mass is calculated. As an Input for the discrete laminate generation, the ply counts, the desired thickness and also design rules and manufacturing criteria are taken into account. As design rules, restriction on the ply counts like minimum and maximum percentage of ply direction, maximum adjacent plies and also sublaminates for inner and outer mould or in the symmetry plane can be defined. The default design and manufacturing rules in the module for composite parts are:

- Symmetric laminates

- Balanced laminates

- not more than four adjacent plies of the same orientation

- $\quad$ add a $45^{\circ}$-ply every sixth ply

- a minimum of $10 \%$ plies of $0^{\circ}$ and $90^{\circ}$ and $20 \%$ of $45^{\circ}$ plies in each component

- a maximum value of $70 \% 0^{\circ}$ and $90^{\circ}$ plies, $80 \%$ of $45^{\circ}$ plies in each component while considering a minimum ply share in each direction

- the first and last three layers of a composite skin are $+45^{\circ} / 90^{\circ} / 45^{\circ}$

- for stiffener webs the first and last layers are $+45^{\circ} /-45^{\circ}$

The first and last layers are chosen for different reasons. First of all, continuous inner and outer plies are guaranteed with these rules. This is desired for reasons of manufacturability, protection of inner layers from moisture, etc. The $45^{\circ}$-layers are shifted outwards for better impact behavior of the laminate, and furthermore, outer $45^{\circ}$ layers are better suited for drilling if this is required for assembly process or repair.

The calculated designs are used to perform an update of the FE-model properties. During that update the stiffness matrix of each component is calculated by HyperSizer and the property entry in the FE input file is overwritten with the new calculated panel stiffness. For Nastran, each property of a stiffened panel is replaced by a PSHELL ${ }^{12}$ property with three MAT2 ${ }^{12}$ cards, representing the $\mathrm{A}, \mathrm{B}$, and $\mathrm{D}$-matrix of the panel. Again this is independent from the availability of discretely modelled stiffeners. If spar caps are modelled, the bar properties are also updated. A new linear static calculation is performed and the ne new FE results are again imported into HyperSizer and the panels are recalculated.

The convergence of the optimization is evaluated by the change of total mass of the model, comparing the mass between each iteration:

$$
\Delta m=\frac{m_{i}-m_{i-1}}{m_{i-1}} .
$$

The value where convergence is achieved has to be chosen by the user. It is recommended not to set a value that is too strict because of the discrete nature of the optimization problem. For a robust process a default value of $\Delta m_{\text {default }}=0.5 \%$ is chosen. Furthermore it is required that $M o S_{\text {min }}>0$ is valid in each panel. In order to save calculation time it is possible to define a maximum number of iterations.

The steering of the optimization is done by an interface script outside of HyperSizer. The script takes the information from the object model that was used to create the FE file with the model generator and uses them to create the components and assemblies in HyperSizer. Also the panel and beam design concepts are assigned to the components in HyperSizer with this script. If the user has chosen to modify the default design and failure criteria the modification of these values in HyperSizer is done by the interface script before the optimization starts. After each iteration the masses and MoS of the applied failure criteria are evaluated and convergence is checked by this script as well and a FE calculation of the updated model is started. If convergence is achieved the interface script calls the CPACS writer which takes the information about design concepts, composite layups, etc. to update the CPACS file.

\section{Exemplary Application: Design-Study and Optimization of DLR-F18 forward swept wing}

In order to demonstrate the capabilities of the structure optimization module, an optimization of a composite wingbox is performed. The investigated configuration is the DRL-F18 configuration, which is a short range aircraft with a forward-swept wing, a T-Tailplane and rear-mounted engines. This structural layout has been determined in a 
previous internal DLR project "LamAiR"; hence the position of front spar, rear spar, and ribs is fixed for this optimization. The wing is shown in Figure 7. The spar coordinates are kept constant along the wing span. The front spar is located at a relative profile coordinate of $15 \%$; the rear spar is located at $60 \%$ of the chord. The ribs are normal to free stream in the center wingbox while they are rotated by approximately $5^{\circ}$ to the aircraft longitudinal axis in the outer wing because of its dihedral. Overall there are 27 rib bays, three in the center wing and 24 in the outer wing, all capable of being fuelled. The fuel loads are considered in the applied load cases. After the last rib, a simple cap is used at the wing tip instead of a winglet.

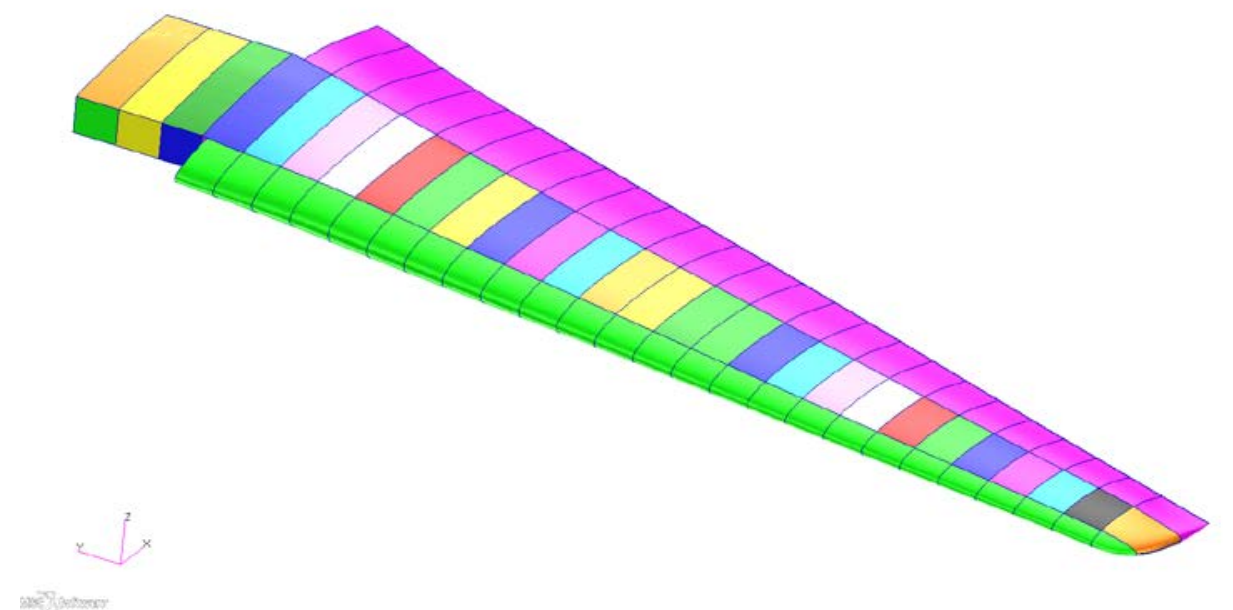

Figure 7: DLR-F18 wing

Not defined in the picked model are the type of stiffeners used in the wing covers and also their pitch. For this optimization, a constant stiffener pitch in spanwise direction of $\Delta s=200 \mathrm{~mm}$ is assumed. Two optimization runs are performed to show the differences in weight, deformation and panel layout and their relation to different stiffening design concepts. In a first one, I-stiffeners are used in the wing covers and in a second one; they are replaced by Tee-stiffeners. Leading and trailing edge are no optimization regions in this scenario. The applied loadcases are listed in Table 2.

Table 2: Applied loadcases to the DLR-F18 wing

\begin{tabular}{lccccc}
\hline CASE & Altitude $[\mathrm{m}]$ & $\mathrm{n}_{\mathrm{z}}$ & Mach & $\mathrm{m}_{\mathrm{F}}[\mathrm{kg}]$ & Fuel in rib bay \\
\hline 1g Cruise & 10668 & +1 & 0.78 & 16100 & $5-27$ \\
3.44g Maneuver & 0 & +3.44 & 0.67 & 706 & $24-27$ \\
-1g Maneuver & 0 & -1 & 0.67 & 17300 & 4.27 \\
\hline
\end{tabular}

The aerodynamic loads are applied as pressure distributions coming from CFD calculations. The CFD results were post processed so that the CFD and FE-mesh were not required to have an identical mesh. The fuel loads are applied by the Nastran "Total Loads" concept to the lower wing cover. The secondary masses were smeared in the center of the front and rear spar panels. Inertia loads of the primary structure are recalculated in each iteration using the NASTRAN card "GRAV" "2. Landing gear loads are not applied because they are unknown at this point.

The optimization regions for this study were the upper and lower wing cover, front and rear spar and the ribs. Each color in Figure 7 represents an optimization region; the leading and trailing edge are .excluded from optimization. Nevertheless they are represented in the FE model for loads introduction. For all components a composite material, a Hexcel T800 / M21 state-of-the-art system is used. As mentioned previously, different stiffener types are used in the wing covers for this design study: Tee- and I-stiffeners. For the ribs and spars the design concept remains the same in both optimizations: unstiffened panels for the front and rear spar and Teestiffened panels for the ribs. The applied failure criteria and layup rules are the default settings described previously in this paper (see Table 1).

The applied convergence criteria is $\Delta m=0.5 \%$. Additional calculations with a more strict criteria $(\Delta m=$ $0.05 \%$.) were also performed Instead of four iterations, nine respectively ten iterations were necessary to achieve convergence, not showing significant differences in mass in structural design. For this application a $0.5 \%$ change is wingbox mass is sufficient as convergence criterion. In Figure 8 the mass convergence plot is shown. In each 
iteration one Nastran and one HyperSizer call was performed, where HyperSizer recalculated the design for all components depending on the new inner loads. Both configurations converged in four iterations, while no negative MoS were detected in all iterations. Nevertheless the designs of the wing covers, and as a result the wingbox stiffness, are present. The results are also presented.

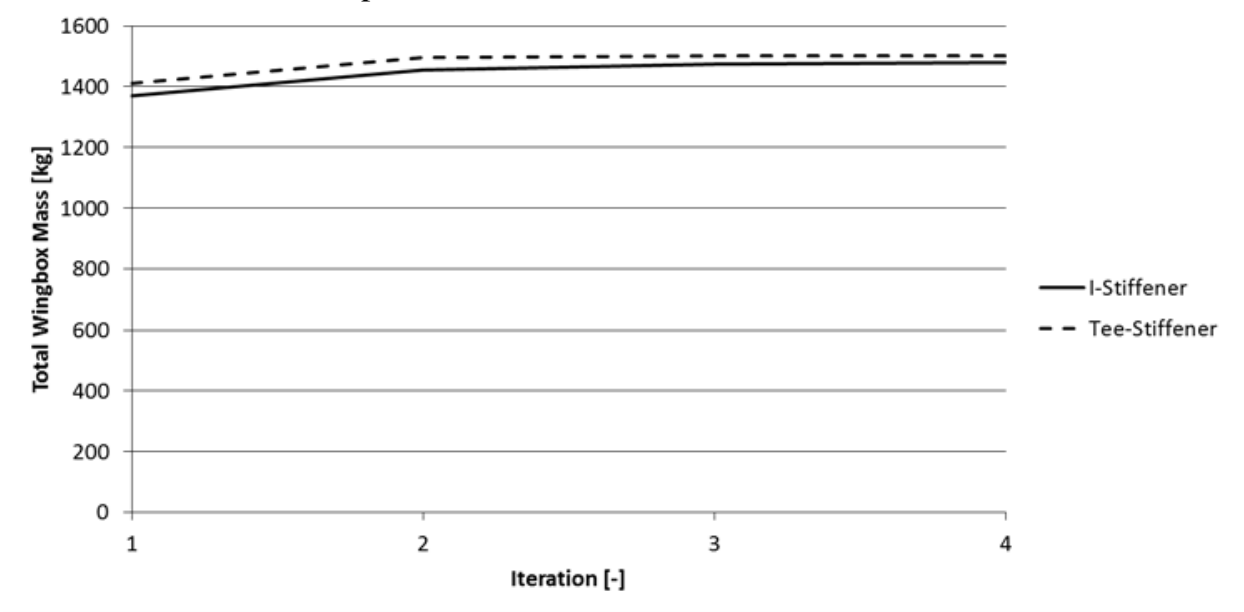

Figure 8: Mass Convergence for LamAiR Example

\section{A. Weight comparison}

The final weight breakdown for the wingbox assemblies is shown in Table 3.

Table 3: Optimized Wingbox Weight Breakdown Comparison

\begin{tabular}{c|c|c}
\hline Assembly & I-Stiffener Mass [kg] & Tee-Stiffener Mass [kg] \\
\hline Upper Cover (skin + stiffener) & 538.67 & 568.88 \\
Lower Cover (skin + stiffener) & 528.96 & 536.35 \\
Front Spar (web) & 116.52 & 109.28 \\
Rear Spar (web) & 155.52 & 148.55 \\
Ribs & 140.20 & 138.59 \\
\hline Total & 1479.87 & 1501.65
\end{tabular}

The wing using Tee-stiffener is $1.5 \%$ heavier than the I-stiffener wingbox. While the wing covers with I-stiffener are lighter than the Tee-stiffener panels, it is the other way around for the related spars. The weight of the wingbox ribs do not significantly change in both configurations.

\section{B. Wingbox Stiffness Comparison}

In Figure 9 and Figure 10 the bending and twist of both configurations in cruise flight is shown. The vertical displacement of the wingbox is fixed at the wing-fuselage-interface, so the diagrams only show the deformation of the outer wingbox. A deformation of the center wingbox (=inner wing) is not present due to the boundary conditions of the wingbox. 


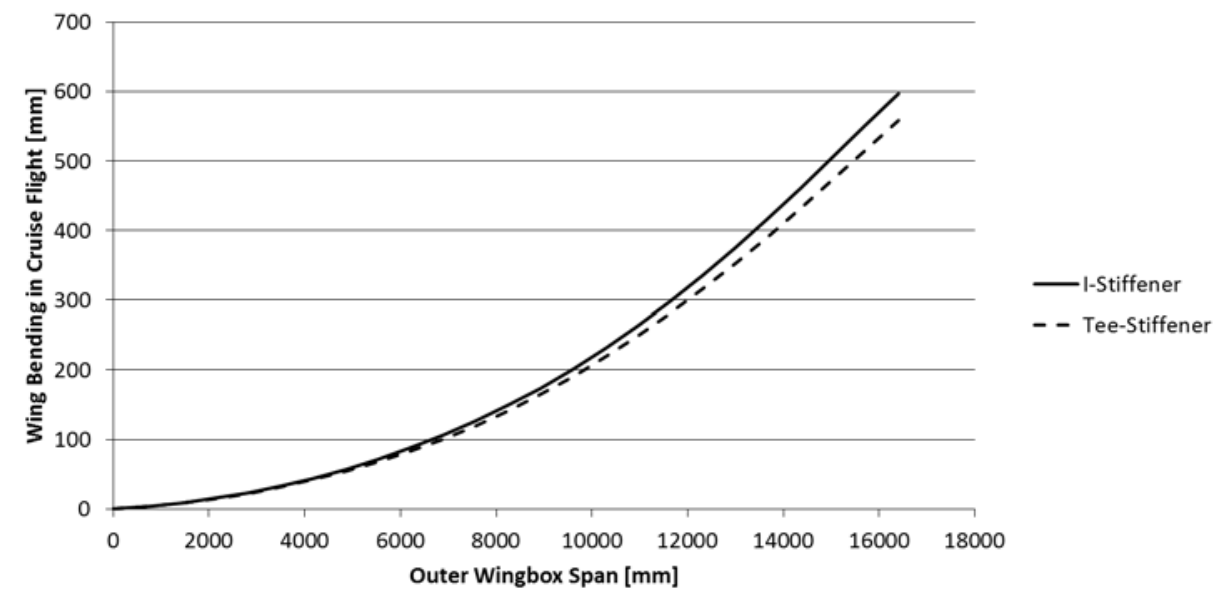

Figure 9: Comparison of Wing Bending in Cruise Flight

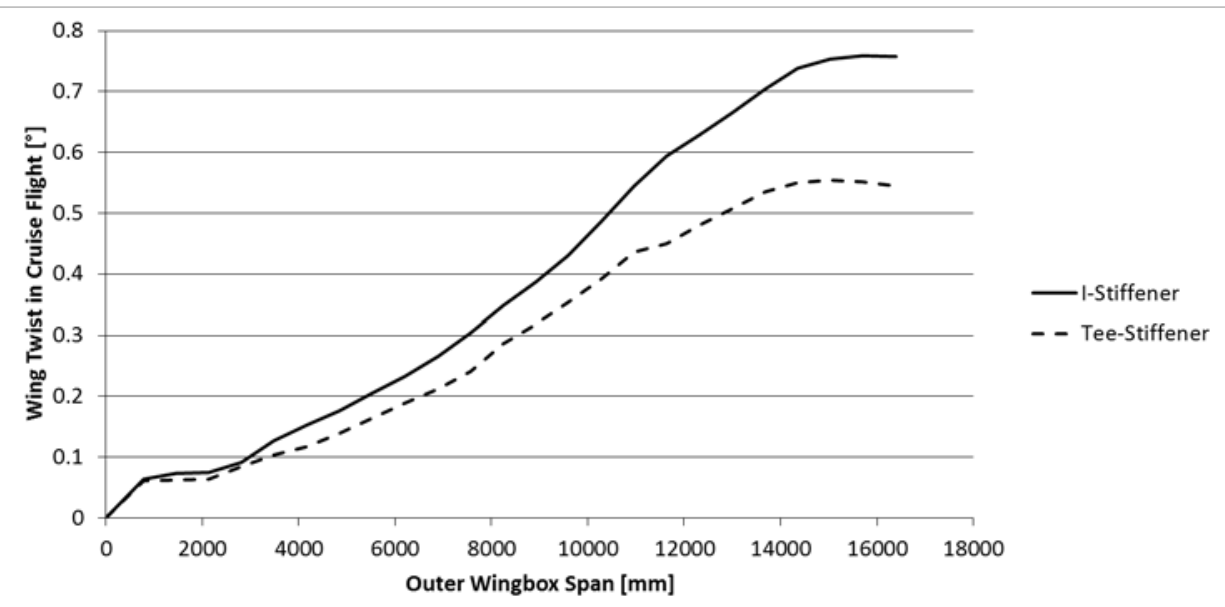

Figure 10: Comparison of Wing Twist in Cruise Flight

Evaluating Figure 9 and Figure 10, it can be seen, that the wing using I-Stiffeners in the wing covers is globally less stiff, especially concerning torsional stiffness. This occurs even though both wings are calculated with the same set of requirements and the same material is used. For this design study, the influence of the stiffener type on the mass is less significant than its influence on the wingbox deformation. The Tee-stiffener wing shows $7 \%$ less vertical displacement and $28 \%$ less twist at the wing tip. This information is important, especially for processes with aero-structure coupled calculations, where the calculated pressure distributions will be different with different wing deformations and therefore, different loads will be applied on the structure. In addition, the difference in wing twist is important for configurations with laminar wings, were the adherence of wingbox twist boundaries is important in order to enable laminar flow.

\section{Design Evaluation}

In order to explain the differences in stiffness, a brief evaluation of the stiffened panel design result is performed. Exemplary, the skin thickness and stiffener design of the upper cover is evaluated. The skin and stiffener web thickness for both upper covers is shown in Figure 11. It can be seen that the skin thickness is almost identical for both wing boxes, but the thickness of the stiffener web is significantly different. Especially in the first half of the wingbox the web thickness of the Tee-stiffener is up to 50\% higher, while the stiffener height does not differ. 


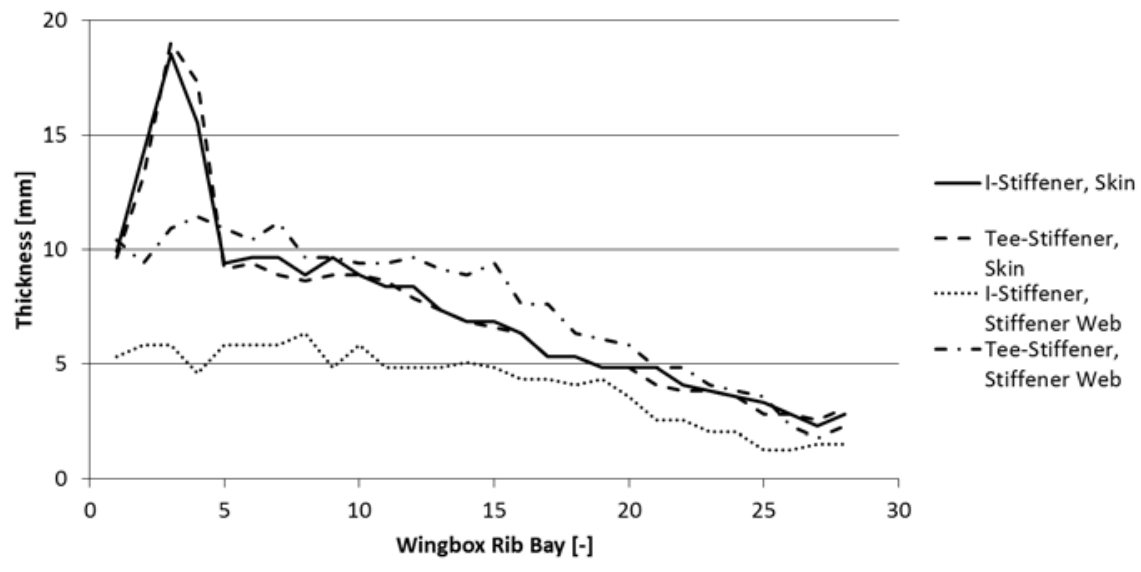

Figure 11: Thickness of Skin and Stiffener Web of Wing Upper Cover

Another interesting fact is the dimensioning failure criteria of the upper cover panels. This distribution is listed in Table 4. In the I-stiffened upper cover, no component is dimensioned by global buckling, while this is dimensioning for some of the Tee-stiffened panels. Therefor more panels are dimensioned by local buckling if Istiffeners are used. The mass of the upper cover components dimensioned by strength is approximately $40 \%$ for both stiffener types.

Table 4: Mass Breakdown of wing upper cover by failure category

\begin{tabular}{|c|c|c|c|c|}
\cline { 3 - 5 } \multicolumn{2}{c|}{} & Strength & Buckling & Local Buckling $^{6}$ \\
\hline Mass from Failure & I-Stiffener & 216.06 & 0.00 & 322.61 \\
Category [kg] & Tee-Stiffener & 227.76 & 99.01 & 242.11 \\
\hline Percental Share of Mass & I-Stiffener & 40.11 & 0.00 & 59.89 \\
from Failure Category [\%] & Tee-Stiffener & 40.04 & 17.40 & 42.56 \\
\hline
\end{tabular}

Evaluating the stiffener type and the mass breakdown from Table 4 it can be stated that the I-stiffener generate a high local bending stiffness preventing the upper cover panels from global buckling. On the other hand the longitudinal stiffness is smaller compared to the Tee-stiffener so more panel are dimensioned by local buckling. This reduced longitudinal stiffness could be one of the reasons for the higher twist. Furthermore the foot of the Teestiffener is wider and thicker than the I-stiffener one so this additional material, with a bigger distance from the wingbox neutral axis, increases bending and torsional stiffness. As a last point, the additional flange of the Istiffener helps to prevent the stiffener web from local buckling. On the other hand it makes the I-stiffener less effective if it comes to global wingbox stiffness because more material is taken away from the stiffener areas with huge distance to the neutral axis.

\section{Conclusion}

In the presented paper, a new structural design and optimization module for the usage in MDO applications was presented. The module is capable to size and optimize composite and metallic wing boxes with respect to a wide range of failure criteria of stiffened panels. The DLR-F18 wing was used to demonstrate the capabilities of the software comparing the influence of two different stringer concepts in the wing upper and lower cover on mass and structural behavior of the wing. It could be shown that with I-stiffened panels it is possible to achieve a lighter wing, which is also less stiff. Hereby it was proven that a different structural design concept can lead to different structural mass and different wingbox stiffnesses. If the deformation of a wing cannot be neglected design concepts must be considered in an early design phase. Examples are laminar wings where the wing twist in cruise flight is often controlled for a better performance of the aerodynamical profiles. Overall, a more realistic wing was calculated, compared to previous projects.

\footnotetext{
${ }^{6}$ Crippling Failure is part of Failure Category „Local Buckling“
} 
The module used for this paper is still in development and will be in future. More criteria or sub-modules, i.e. for twist control will be implemented. It will also be possible to use the module for the optimization of a fuselage or a rotor blade for wind energy stations.

\section{Acknowledgments}

The work for this module involved the time and expertise of several people not mentioned in this paper. Special thanks goes to people of Collier Research making HyperSizer program features available for DLR by their own program object model and for their great support for several years. Furthermore we would like to thank our colleagues in the structure mechanics department of our institute for the good cooperation with the model generator and the connection of the model generators object model with the design module.

\section{References}

${ }^{1}$ Sobieszczanski-Sobieski, J. and Hafka, R. T., „Multidisciplinary Aerospace Design Optimization: Survey of Recent Developments“, Structural Optimization, Vol. 3, No. 14, 1997, pp. 1-23

${ }^{2}$ DeBlois, A. and Abdo, M., "Multi-Fidelity Multidisciplinary Design Optimization of Metallic and Composite Regional Business Jets”, $13^{\text {th }}$ AIAA/ISSMO Multidisciplinary Analysis Optimization Conference, Fort Worth, 2010

${ }^{3}$ Schuhmacher, G. et all, „Multidisciplinary Airframe Design Optimization“, $28^{\text {th }}$ International Congress of the Aeronautical Sciences ICAS, 2012

${ }^{4}$ Ronzheimer, A. and Natter, F. J. and Brezillon, J., “Aircraft Wing Optimization Using High Fidelity Closely Coupled CFD and CSM Methods”, $13^{\text {th }}$ AIAA/ISSMO Multidisciplinary Analysis Optimization Conference, Fort Worth, 2010

${ }^{5}$ HyperSizer, Software Package, Ver. 7.0.35, Collier Research Corporation, Newport News, 2014

${ }^{6}$ Kroll, N. et all. "Digital-X Projektplan, “ DLR, Braunschweig, 2012

${ }^{7}$ Führer, T. et all, “Digital-X, Report Milestone 4.2.1”, DLR IB 131-2012/72, 2012

${ }^{8}$ Bruhn, E., Analysis and Design of Flight Vehicle Structures. Jacobs Publishing, Inc., 1973

${ }^{9} \mathrm{Niu}$, M. C., Airframe Structural Design, Conmilit Press Ltd., Hongkong, 1997

${ }^{10}$ US Department of Defense MIL-HDBK-17-3F, Composite Materials Handbook, Volume 3. Polymer Matrix Composites Materials Usage, Design, and Analysis, 2002

${ }^{11}$ Collier Research, HyperSizer Documentation, Collier Research Corporation, Newport News, 2014

${ }^{12}$ MSC Software, MSC Nastran 2013.1 Documentation, Quick Reference Guide, MSC Software, Newport Beach, 2013

${ }^{13}$ Huerlimann, F. et al, „Investigation of local load introduction methods in aircraft pre-design“, Aerospace Science and Technology, Vol. 21, 2012, pp. 31-40 